\title{
Caractéristiques des savoirs des patients et liens avec leurs pouvoirs d'action : implication pour la formation médicale
}

\section{O. Gross ${ }^{1} \&$ R. Gagnayre ${ }^{1}$}

${ }^{1}$ Laboratoire Éducations et Pratiques de santé (EA3412), Université Paris 13 - Sorbonne Paris Cité

Résumé -Dans le contexte de la démocratie en santé, de nombreux espaces participatifs sont ouverts aux patients et à leurs proches, pour qu'ils complètent de leur perspective celle des professionnels de santé. Une série de recherches a été mobilisée afin de contribuer à caractériser cette perspective, à partir de l'analyse des savoirs des patients, de leur processus d'émergence, de leur valeur et de leur utilité. Cinq savoirs, parfois imbriqués, ont été identifiés : des savoirs expérientiels implicites qui visent une auto-adaptation, des savoirs expérientiels explicites qui visent une hétéro-adaptation, des savoirs situés qui permettent aux patients de se constituer en communauté épistémique et des savoirs savants qui soutiennent les précédents et peuvent leur permettre d'accéder à des savoirs experts. La taxinomie proposée contribue aux travaux conceptuels sur les savoirs profanes. Elle propose des liens entre les savoirs et les pouvoirs y afférents et permet de repérer quel savoir solliciter, dans quel dispositif pédagogique.

Mots clés - Engagement des patients, savoirs expérientiels, savoirs situés, savoirs savants, savoirs experts, pouvoir d'agir 


\section{Introduction}

L'engagement des patients en collaboration avec les professionnels de santé dans le système de santé français est un phénomène en croissance et particulièrement protéiforme (Gross, 2017). Depuis la loi de 2002 relative aux droits des malades et à la qualité du système de santé, 20000 mandats sont destinés aux usagers du système de santé afin que, par l'intermédiaire de leurs représentants élus, ils participent à la politique de qualité et de sécurité des soins des hôpitaux. Dans la lignée du modèle de Montréal (Pomey et al. 2015a) leur engagement tend à s'étendre au-delà du cadre prévu initialement par cette loi.

En France, leur participation à l'éducation thérapeutique des malades, ou à leur accompagnement, a été vivement encouragée. Ainsi, la loi portant réforme de l'hôpital et relative aux patients, à la santé et aux territoires (2009), plus connue sous l'expression « Hôpital, patients, santé et territoire » a fait émerger la figure de patients co-éducateurs de leurs pairs dans l'éducation thérapeutique des patients. Et des postes de coordination commencent à être créés à leur intention et certains hôpitaux, comme c'est le cas des hospices civils de Lyon, recrutent des usagers comme coordonnateurs du développement du partenariat avec les patients. De même, dans le cadre d'une série d'expérimentations, des anciens usagers de la psychiatrie sont employés par des services psychiatriques pour participer aux soins des patients qui y sont suivis (Demailly, 2015). Émergent également des «navigateurs de santé pairs » dont la fonction est d'accompagner, parfois au quotidien, les personnes en difficulté avec le système de santé, tels les «compagnons maladies rares »dont l'activité est financée actuellement par la Direction Générale de la Santé dans le cadre d'un programme expérimental d'accompagnement à l'autonomie en santé en lien avec l'article 92 de la dernière loi de santé publique. De plus, de manière plus ou moins formelle, des patients sont également associés aux différentes phases des recherches, dans le cadre de recherches dites collaboratives. Ce type de recherche commence tout juste à être encouragé en France (notamment dans l'axe 4 de la stratégie nationale de santé) alors que dans certains pays, comme au Royaume-Uni, les financements des recherches publiques sont conditionnés au choix de cette méthodologie de recherche (NHS, 2014). Enfin, émergent des patientsenseignants (Gross et al. 2017a) dont l'intégration dans la formation des professionnels de santé et du social découle des recommandations émises dans le rapport «l'an II pour la démocratie sanitaire »(Compagnon, Ghadi, 2014), recommandations récemment reprises dans la stratégie MaSanté2022.

Cette institutionnalisation des patients comme acteurs du système de santé peut sembler être un contrepoint au pouvoir des professionnels de santé. Mais l'engagement des patients n'est pas une prise de pouvoir des patients puisque, au contraire, ce qui leur est demandé - et ce qui fait sens pour eux-c'est de compléter de leur perspective, et parfois de contrebalancer, celle des professionnels. Aussi, différentes institutions et un nombre croissant d'acteurs sont de plus en plus convaincus de l'intérêt de collaborer avec les patients pour améliorer certaines actions de santé. Pour ces derniers, « la participation des patients et citoyens est devenue un enjeu sociétal et politique pour des raisons démocratiques, morales et éthiques, mais aussi pratiques »(Druais, 2015). Autrement dit, au-delà des raisons morales qui renvoient au fait 
qu'il est juste que des personnes concernées par une problématique participent à sa résolution, ils considèrent que si leur participation vaut, c'est qu'elle apporte des éclairages uniques. À l'origine, de ces éclairages, on reconnaît des savoirs expérientiels (Jouet, Flora, Las Vergnas, 2010) et il est souvent dit qu'ils sont une richesse inestimable, une source d'information précieuse, pour compléter les savoirs des professionnels de santé (Pomey et al. 2015a, 2015b) dans une perspective de croisements des savoirs (Galvani, 1999).

Toutefois même dans un pays qui reconnaît la valeur de l'expérience au point de la diplômer via la procédure de validation des acquis de l'expérience, la reconnaissance des savoirs expérientiels des patients reste compliquée. Peut-être que leur extrême inter-variabilité (d'Arripe, Routier, 2013), ne plaide pas pour leur assise, donc pour leur intérêt. De plus, la science s'étant construite contre le sens commun, l'octroi de savoirs seulement expérientiels aux patients pourrait expliquer l'effet paradoxal qui conduit à les discréditer ou à les cantonner à un statut subalterne.

Référer les savoirs des patients aux seuls savoirs expérientiels n'est jamais remis en question, pas même par les patients eux-mêmes qui pour certains revendiquent être des «experts de l'expérience » (Mac Laughlin, 2009). Les savoirs expérientiels semblent leur permettre de se distinguer des professionnels de santé et de se légitimer. Mais, attribuer des savoirs expérientiels aux seuls malades, surtout comme c'est le cas pour les différencier de ceux des professionnels de santé, ne résiste pas à l'analyse compte-tenu que la pratique de ces derniers est aussi conditionnée par leurs propres savoirs expérientiels qui renvoient à leur expérience de soignants (Sackett et al., 1996).

En outre, les patients sont nombreux à interagir avec leurs pairs au sein de communautés virtuelles ou physiques (Akrich, Meadel, 2010), à se cultiver en santé, notamment sur Internet (Hardey, 1999; Romeyer, 2008) et à se former au sein d'espaces formels, y compris universitaires (Santi, 2016).

Est-ce que les savoirs acquis dans ces derniers contextes relèvent encore vraiment de l'expérientiel ? Où commence et où s'arrête l'expérience ? Ou à quel moment l'expérience qui qualifie certains savoirs cède le pas à d'autres types de savoirs ?

À l'heure où se multiplient les propositions de participation des patients (notamment dans l'éducation thérapeutique, l'accompagnement, la médiation, la recherche et la formation médicale et paramédicale), il devient nécessaire de caractériser plus avant leurs savoirs. En effet, un des enjeux actuels porte sur le fait de recruter le bon profil de patient au bon endroit, c'est à dire de lui proposer l'activité dans laquelle il sera le plus à l'aise, donc le plus utile. Différentes options se présentent. Leur identification est actuellement soumise à leur statut (membre associatif ou pas, représentant d'usager ou pas), ou à leurs compétences réelles ou supposées à partir de référentiels établis en amont de leur recrutement (Pomey et al. 2015b). II y a aussi une certaine tension entre le fait de vouloir recruter des patients authentiques (Towle, 2016), définis comme des patients qui ressemblent à ceux vus au quotidien au cours de l'exercice médical, et des patients plus engagés, plus efficaces, mais 
soupçonnés de porter une voix plus militante, et moins représentative de tous les patients. Ces questions se posent avec la même urgence dans d'autres espaces participatifs que la santé (Blondiaux, 2008, Gourgues et al. 2013). Aussi cet article vise à contribuer à dresser une épistémologie des savoirs dont les profanes - ici les patients - sont vecteurs afin d'interroger leur engagement au regard de leurs types de savoirs, sachant qu'il n'y a pas de pratiques, donc de compétences, sans savoirs (Perrenoud, 1998).

\section{I- Méthodologie}

Pour donner un cadre descriptif aux savoirs des patients, pour cheminer vers une épistémologie fondée sur leur interaction avec l'environnement et sur les gains en pouvoir qui en résultent, nous nous appuyons, sur les résultats de quatre recherches qualitatives adossées au paradigme socioconstructiviste menées depuis une dizaine d'année :

- La première recherche portait sur la figure des patients experts. Elle visait à partir d'entretiens compréhensifs réalisés auprès de douze personnes à en déterminer les caractéristiques, notamment sur le plan de leurs savoirs, compétences et motivation (Gross, Gagnayre, 2014).

- La seconde portait sur vingt patients-enseignants recrutés notamment pour leur culture en santé et leur engagement associatif pour enseigner au sein d'un département de médecine générale. Son objectif était de caractériser à partir d'observations non participantes leur perspective telle qu'elle s'exprimait dans des groupes d'échanges de pratiques réflexives entre internes (Gross et al. 2017a, Gross et al. 2017b).

- La troisième a permis, à partir d'une revue de littérature d'identifier l'apport et les profils des patients co-chercheurs dans les recherches-actions en éducation thérapeutique (Gross, de Andrade, Gagnayre, 2017c).

- La quatrième interrogeait le caractère émancipateur d'une formation destinée à des patients au départ peu qualifiés, pour qu'ils deviennent éducateurs de leurs pairs au sein de programmes d'éducation thérapeutique (Gross et al. 2016).

- Enfin, nos observations participantes durant plusieurs années au sein de Commissions des Usagers (CDU) dans un hôpital de l'Assistance Publique-Hôpitaux de Paris, ainsi qu'au sein d'associations de malades, ont complété ces différents terrains ${ }^{1}$.

Les matériaux des entretiens et des observations avaient été conservés, soit au total douze entretiens et environ trois cents heures d'observations. Une nouvelle analyse a été faite afin d'extraire de ces données le corpus propre aux savoirs des patients. L'ensemble ainsi constitué ne correspond pas exactement au projet initial de ces recherches. La diversité des terrains de recherche mobilisant différentes situations et différents profils de patients garantit une validité qualitative à l'analyse rétroactive qui a porté sur ce corpus.

\footnotetext{
${ }^{1}$ Le premier auteur de cet article est chercheure en sciences de l'éducation et par ailleurs elle est représentante d'usagers au sein d'un hôpital au nom de l'association agréée dont elle est présidente.
} 
Dans le cadre de cette nouvelle recherche, l'analyse a été reproduite et croisée avec les travaux propres aux savoirs et à la formation de soi (Piaget, 1970, 1974, ; Kolb, 1984 ; Haraway, 1991 ; Galvani, 1999). Elle a été adossée au modèle tri-polaire de Pineau (1986) constitué de l'auto-formation, de l'hétéro-formation et de l'éco-formation qui interagissent pour produire le sujet connaissant. Ces trois dimensions ont servi de grille d'analyse pour organiser les modalités d'émergence des savoirs des patients. Ces derniers ont fait l'objet d'une analyse thématique réalisée par le chercheur principal depuis longtemps exposé au phénomène et l'objet d'une supervision par le second chercheur.

Pour chaque expression d'un savoir, différentes questions ont guidé l'analyse : à quoi réfèrent-ils ? qu'est ce qui fonde leur valeur ? à quoi servent-ils ? Cette dernière question provenant du postulat de l'existence d'un lien entre les savoirs et le pouvoir d'action (Stehr, 2000).

\section{Résultats}

Cinq types distincts de savoirs pouvant être acquis et véhiculés par les patients ont été identifiés (par «patient», nous entendons aussi bien malade que proche de malade). Pour asseoir la validité externe de ces résultats, ceux-ci ont été présentés dans différents contextes (des enseignements et des communications dans des contextes associatifs) à des groupes de patients qui s'y sont reconnus et se les sont appropriés facilement.

\section{Les savoirs expérientiels implicites des patients : pour un pouvoir d'agir sur soi fondé sur les savoirs de l'épreuve}

La maladie est vue comme une occasion d'apprentissages (Tourette-Turgis, 2015). Les savoirs que l'on y acquiert sont dits d'ordre expérientiel, en référence aux savoirs identifiés dans le cadre de l'andragogie (Kolb, 1984). Leur processus de construction se caractérise par des épisodes d'autoformation basés sur des expériences concrètes que la répétition des faits met à l'épreuve pour en tirer du sens, des hypothèses, des règles, puis enfin des actions adaptées. Il s'agit donc de savoirs dont la visée est l'action juste, considérée comme telle à partir de ce qui fonctionne (Piaget, 1970). En ce sens, il s'agit de savoirs empiriques, fonctionnels, qui permettent une emprise sur le monde, tout en étant idéalement sans cesse remodelé par lui. Il est dit qu'ils renvoient à l'expérience intime qui lie un malade particulier à sa maladie particulière (Barrier, 2012), à sa propre trajectoire de soins et aux répercussions de ses problèmes sur sa vie personnelle (Pomey et al., 2015a). Ces éléments confirment que ces savoirs ne peuvent avoir la prétention de s'inscrire dans une perspective généralisante. Mais ils n'épuisent pas la question des liens entre ces expériences et les savoirs-pouvoirs qui en découlent. D'après nos résultats, les savoirs expérientiels des malades, qui renvoient à des savoirs de l'épreuve, sont liés à leur expérience :

- de la vulnérabilité. Pour Dewey (1938), l'expérience est un événement social. Dans le contexte de la maladie, l'expérience n'est qu'en partie un événement social car elle est aussi intime : la maladie est sans conteste l'épreuve par excellence dont il est inutile de rappeler ici à quel point elle relève de la plus tragique angoisse existentielle, 
- de la vie quotidienne avec la maladie. Dans ce domaine, les savoirs peuvent être réflexifs et servir à l'agir, et notamment à l'agir au quotidien pour sa santé. En effet, si on peut savoir sans savoir y faire, pour réussir il faut savoir comment on réussit (Le Boterf, 2003). Cela est encore plus vrai quand il s'agit de réussir souvent, ce qui est le lot des malades chroniques. Le métier de patient (Tourette-Turgis, 2015) convoque un processus de métacognition qui sert aux patients à devenir conscient de ce qu'ils savent pour pouvoir prendre soin d'eux-mêmes, et servir leurs compétences d'auto soins ou d'adaptation à la maladie (d'Ivernois, Gagnayre, 2001).

De plus, toujours d'après nos résultats, leurs savoirs vont au-delà de l'expérience intime avec la maladie puisqu'ils renvoient également :

- au parcours de soins. Les savoirs expérientiels qui portent sur le parcours de soins s'adossent à leur vécu de l'effectivité des parcours de soins, tant il est vrai que l'accès aux soins et aux droits est marqué à la fois par des difficultés qui ne sont pas éprouvées par ceux qui ne le vivent pas, et par la nécessité de développer des compétences pour s'orienter où il le faut quand il le faut,

- à la relation de soins. Les savoirs des patients qui renvoient aux relations de soins, concernent leur perception de cette relation. Émanant de ceux qui au sein cette relation sont en position de demande, ces savoirs, comme les précédents leur sont éminemment spécifiques.

Ces savoirs expérientiels leuront permis aux patients d'opérer un tri entre ce qui fonctionne et ce qui ne fonctionne pas pour eux. Dans certains cas, ils restent implicites, c'est à dire qu'ils sont difficilement transmissibles et valorisables car tous les patients n'ont pas repéré parmi leurs savoirs ce qui vaut et ce qui ne vaut pas pour autrui, ni ce qui en fait un objet spécifique au regard des savoirs des professionnels. Ne les ayant pas soumis à l'épreuve de la généralisation, ils sont donc dans l'incapacité d'en discerner la valeur, et encore moins pour qui et dans quel contexte. Par conséquent, ce type de savoirs a besoin d'un soutien maïeutique pour émerger et être repéré. Ce sera l'objet d'entretiens, de questionnaires, ou encore de groupes de paroles sous forme de discussions portant sur le vécu en lien avec la maladie et sur des analyses de pratiques.

Un retour sur les expériences, notamment guidé lors de groupes de parole, peut faciliter la prise de recul nécessaire pour que les participants prennent conscience de leurs savoirs expérientiels. Ce mode cognitif est dit réfléchi car l'analyse permet de comprendre rétrospectivement l'expérience (Chevrier, Charbonneau, 2000) et de lui donner du sens (Tardieu, 2015). Ils permettent aussi de faire émerger le général derrière le particulier, ou encore la règle qui doit servir à guider l'action. À ces conditions, ces savoirs peuvent devenir explicites. 


\section{Les savoirs expérientiels explicites des patients : pour un pouvoir d'agir sur autrui fondé sur le kairos}

Au contraire des savoirs expérientiels implicites, nos résultats montrent que les savoirs expérientiels explicites ne visent pas l'auto-adaptation mais l'hétéro-adaptation, c'est à dire l'influence sur autrui. Ce qui nécessite des savoir-faire spécifiques et le recours à des savoirs en partie autres.

Les patients ayant développé des savoirs expérientiels explicites sont en capacité d'exprimer leurs savoirs - ou certains d'entre eux - de la bonne manière, au bon moment, à la bonne personne. Le discernement du moment opportun pour agir de la bonne manière renvoie à la notion aristotélicienne de kairos. Le kairos, ainsi que leur pouvoir d'agir, caractérisent ce profil de patients. Pour ce qui est du pouvoir d'agir, dans les situations dont il est ici question, il renvoie en particulier à une de ses composantes qui est l'influence sur autrui (Bravo, 2015). Il s'agit donc de la compétence à influer dans le sens escompté sur autrui. L'émotion liée à la vulnérabilité est contenue, apprivoisée, pour que les savoirs expérientiels explicites qui portent sur les évènements passés soient traduits dans un ici et maintenant : " il faut traverser la vaste carrière du temps pour arriver au centre de l'occasion" (Baltazar Gracián, cité par Jankelevich, 1978). Il s'agit de faire le tri entre tous les savoirs expérientiels pour mobiliser le plus utile pour atteindre l'objectif fixé. Cela passe par transmettre ses savoirs de manière efficace et utile, ou par les exprimer de manière à être écouté. Le kairos n'est rien sans le savoir qui permet de le capter, aussi, comme évoqué, il ne s'agit pas seulement d'habilités communicationnelles, mais de discernement. La métacognition de ce groupe de patients ne porte pas seulement sur la conscience de leurs savoirs, mais aussi sur la conscience de leur environnement. Cela demande d'avoir les mots pour le dire dans les schèmes d'intelligibilité de leurs interlocuteurs. Et cela peut demander une certaine culture en santé en particulier quand ces interlocuteurs sont des professionnels de santé et qu'il faut à la fois se faire comprendre d'eux, avoir une idée de ce qui leur paraît pertinent et de ce qu'ils sont prêts à entendre.

«Quand on veut communiquer avec des personnes, il faut pouvoir rentrer dans leur sphère de repères » (G., patient-expert, recherche 1)

«Vous faites un métier difficile. Vous êtes confrontés à la souffrance, à la frustration. Les gens vont mal et attendent beaucoup de vous. Et il y a des enjeux de vie ou de mort. L'idée ce n'est pas de vous dire ce qu'il faut faire mais de mettre des mots sur ce qui est difficile. Sur le papier on distingue toutes les souffrances, physiques, morales, psychologiques, mais dans la vie tout est groupé » (M., patient-enseignant, recherche 2).

Aussi, les patients qui ont ce type de savoirs peuvent avoir besoin de culture en santé, et en ce cas chercher à l'acquérir de manière autodirigée au sein d'espaces éducatifs formels et/ou informels.

Au sein d'espaces formels tels ceux dédiés à l'éducation thérapeutique ou dans d'autres espaces plus informels comme ceux où prend place l'entraide mutuelle, ces savoirs servent à influencer sur les apprentissages des autres patients pour qu'ils gagnent en qualité de vie ou en libertés de bien-être. Ce processus renvoie à la pair-émulation (Gardien, 2010) qui peut s'exercer d'un pair vers un autre, et parfois réciproquement, quand un individu s'engage pour 
soutenir le pouvoir d'agir d'un autre, ce qui participe rétrospectivement à donner du sens à ses savoirs de l'épreuve.

Ces savoirs sont également nécessaires pour témoigner de parcours de vie auprès des professionnels de santé en formation initiale ou continue. Ils n'en demeurent pas moins des savoirs expérientiels car ces patients puisent toute leur légitimité dans leurs histoires de vie individuelles. Ce sont elles seules qui donnent sens à leurs messages. Selon nous, ces savoirs peuvent être qualifiés d'explicites car ils s'expriment à bon escient et de manière autonome.

À noter toutefois que leur spontanéité n'est pas toujours aussi réelle qu'elle le paraît : le sens du kairos, comme la capacité à influer sur autrui, peuvent avoir besoin d'espaces éducatifs pour émerger et s'exercer. L'élaboration d'autobiographies raisonnées (Desroche, 1990) au sein de ces espaces y contribuent fortement. Ces autobiographies servent alors d'outils pour porter des messages.

« Parler de sa maladie, c'est l'entrée en matière pour rentrer en contact ». (R., patient en formation d'éducation thérapeutique recherche 4)

Sachant que les lieux dédiés à la formation qui visent le développement de ces savoirs doivent rester des espaces de production de savoirs avant que d'être des lieux de transmission de savoirs (Galvani, 2016).

\section{Les savoirs situés des patients : pour un pouvoir d'agir sur le monde fondé sur l'esprit critique}

D'après nos observations, dans les collectifs associatifs, ou au sein de communautés diverses et variées, les patients échangent entre eux sous forme de «dialogues herméneutiques » (Galvani, 2016) au cours desquels ils revisitent leurs savoirs expérientiels. L'expérience collective de l'épreuve de la maladie permet un retour au commun basé sur la conscientisation (Freire, 2001), soit le repérage des situations injustes (Renault, 2004). Partir des expériences collectives, les croiser avec les bonnes pratiques, revisiter les normes avec un regard critique, permet de se forger un point de vue collectif, soit une forme d'épistémè collective.

Les savoirs ainsi produits sont des savoirs situés dont des épistémologues ont montré qu'ils remettaient en cause toute notion d'objectivité portée par une seule partie des acteurs d'une réalité (Haraway, 1991). Pour les patients, comme avant eux pour les autres groupes auprès desquels ces savoirs ont été identifiés, l'identification des savoirs permet de concevoir une action collective, coordonnée, qui vise à transformer le monde pour répondre aux besoins constatés.

«Le principe général, c'est de se dire qu'on veut essayer de faire que les malades soient acteurs de leur maladie, autrement dit, qu'ils ne soient que passifs, pas que patients d'ailleurs, et qu'ils essaient de formuler leurs besoins, leur manière de voir les choses, qu'ils mettent en avant les questions qui sont pas forcément soulevées par les scientifiques, c'est d'être collaboratif, imaginatif, c'est d'aider à faire avancer les choses » (H., président d'association, entretien semi-directif, recherche 1).

Les savoirs situés visent comme les savoirs expérientiels explicites à influer sur le réel, mais 
ils se distinguent de ces derniers à plus d'un titre. D'une part, ils se construisent contre les phénomènes locaux, les épiphénomènes, tout en s'enrichissant à leur aune. Eminemment sociaux, ils sont bien plus stabilisés que les savoirs expérientiels, puisque ce qui les guide, c'est la quête d'unité et l'action coordonnée. De plus, le pouvoir-d'agir communautaire qui en résulte est fondé sur une lecture critique de l'environnement (Alinsky, 1946 ; Freire, 2001).

«On avait la loi la plus restrictive du monde en ce qui concernait la greffe ! Avec un seuil des donneurs vivants qui était un inventaire à la Prévert des gens qui étaient autorisés à donner, par exemple...avec interdiction des dons croisés, avec plein de choses qui étaient extrêmement rétrogrades. On voulait que ça évolue ». (Y., présidente d'association, recherche 1)

Une liberté dogmatique résulte de cette lecture critique : «certains professionnels de santé publique consultés soutiennent que, libre de toute contingence administrative, corporative, politique ou économique, le citoyen, une fois correctement informé par les experts des données probantes, se trouve probablement plus libre dans ses jugements sur l'arbitrage des valeurs en conflits » (Massé, 2005).

« Je ne catégorise pas cet évènement indésirable comme vous. Il ne s'agit pas d'une erreur de médicament mais d'une erreur sur la personne. » M. Représentant d'usager. Observation participante au sein d'une commission des usagers.

D'autre part, ces savoirs ne se manifestent pas de la même manière que les savoirs expérientiels : le passé des patients qui mobilise les savoirs est nié : au contraire de ce que l'on constate dans le cadre des savoirs expérientiels explicites, ils font le moins possible référence à leur histoire personnelle. Ils se posent plutôt en fer de lance de leur communauté dont ils se présentent comme représentatifs puisque leur voix s'élève en son nom pour délivrer un récit socialement construit.

«C'est vraiment cette notion de porte-parole. Je ne me sens pas à l'aise de représenter un groupe, si on n'a pas fait un travail en amont qui consiste à élaborer ensemble quel est le discours, les positions, qu'on veut tenir ». (H., président d'association, recherche 1)

Les patients les plus engagés au niveau collectif ont des savoirs situés. Leur épistémologie commune permet que, de la même manière que les professionnels vont interpréter un événement à partir d'une grille de lecture qui renvoie à leurs logiques, ils y réagissent à partir de leurs propres logiques qui sont stabilisées d'un patient à l'autre. D'après nos résultats (Gross et al. 2017b), ils procèdent par énaction (Varela, Thompson, Rosch, 1993), à partir des valeurs communes qui les animent (la dignité, l'autonomie, l'équité, la participation...).

En ce sens, ils représentent une communauté épistémique dans l'acception qu'en donne Haas (1992) car ils partagent les mêmes savoirs, les mêmes valeurs, les mêmes objectifs. C'est ce qui justifie notamment leurs mandats comme représentants des usagers du système de santé 
puisqu'ils parlent spontanément d'une même voix. Comme nous l'avons constaté à partir de nos observations, toute analyse d'une plainte déposée par un usager par exemple, génèrera les mêmes analyses d'un représentant d'usager à l'autre. Les directions hospitalières ont souvent tendance dans cette situation à chercher à se protéger d'éventuelles actions judiciaires et à estimer que l'émotion des plaignants discrédite leur parole. Les représentants d'usagers vont pour leur part comprendre leur émotion, penser à leur résilience ainsi qu'à l'effectivité des actions correctrices (qui d'ailleurs contribuent à la résilience des plaignants qui sont nombreux à déclarer dans leurs plaintes qu'ils ne font part de leurs déboires que pour qu'ils soient évités à d'autres).

Dans d'autres espaces, ils peuvent réinterroger les savoirs dominants, soit les normes scientifiques ou professionnelles, car les faits ont pour eux une valeur qui prédomine celle des preuves scientifiques. Ainsi, ils excellent dans le rôle de lanceur d'alerte, que ce soit concernant des effets indésirables liés aux traitements, des symptômes non répertoriés, de nouveaux besoins en termes de droits ou d'organisation des soins....

« Je voyais d'autres usagers avec lesquels j'essayais de mettre en commun cette idée d'où ça venait, qu'on devenait de plus en plus maigres avec des trous dans les joues ». (M. patient-expert, recherche 1)

Il peut parfois être utile de soutenir le développement de savoirs situés au sein d'espaces éducatifs. Ainsi, dans le cadre d'un programme de patients-enseignants auprès des internes en médecine générale comme dans celui d'une Licence de sciences sanitaires et sociales destinée aux médiateurs de santé pairs à l'UFR de Santé Médecine et Biologie Humaine (SMBH) de Bobigny-Université Paris 13, il est prévu des temps pour questionner les savoirs expérientiels des patients, pour leur faire revisiter certaines normes afin de faire émerger des construits collectifs et partagés, des prises de position collectives, et de soutenir l'émergence d'une communauté épistémique et de pratiques.

\section{Les savoirs savants et experts des patients : pour un pouvoir d'agir sur les choses, fondé sur les connaissances}

Enfin, dans le contexte facilitant propre à la société du savoir (Unesco, 2005), certains patients, décrits comme des «e-patients » (Ferguson, Frydman, 2004) ou comme des patientsexperts (Gross, Gagnayre, 2015) accèdent à des savoirs savants définis comme des connaissances générales et abstraites (d'Arripe, Routier, 2013). Ces savoirs concernent un domaine de la santé (organisation des soins, droits des malades, développement des médicaments, etc.), une maladie en particulier, ou un groupe de maladies et leurs traitements. Les patients les acquièrent de manière autonome, autodirigée, en particulier lors d'interactions avec des experts et en se documentant. Les savoirs savants des patients n'ont pas forcément de spécificité particulière par rapport aux savoirs véhiculés par d'autres types d'acteurs sociaux, à la limite près que dans le domaine médical, il est rare que des personnes sans culture médicale initiale accèdent pleinement aux savoirs académiques. Néanmoins, cela peut servir leur intuition scientifique, comme cela a été le cas pour K. qui a initié et développé une thérapie médicamenteuse : 
«Je pense que les gens qui sont successful sur le plan de la science, c'est des gens qui font comme moi : qui papillonnent très très loin de la source de la connaissance. Si tous avaient cette ouverture, ce papillonnement... » (K., patient-expert, entretien semidirectif, recherche 1)

Comme les précédents, ces savoirs leur servent à agir, soit de manière individuelle, soit de manière plus collective.

«En 96, c'était...j'étais parmi ceux qui n'étaient pas morts mais je me disais que je ferai toujours partie de ceux qui seraient en première ligne, qui auraient toujours un train de retard par rapport à la population qui viendra après. Je me disais que j'aurai toujours à faire avec l'incertitude et que dans ce contexte, il faudra m'emparer de la dernière étude car je serai toujours à court de stratégies ». (M., patient-expert, entretien semi-directif, recherche 1)

«Il n'y a pas 50000 solutions, en matière de recherche scientifique, il faut aller à la source, parce que, si on est à l'aval de tout ce que les gens produisent, on n'a jamais d'anticipation, on est que suiveurs. Comme ce qui nous intéresse, c'est d'avoir une action politique, qui dit action politique, dit un minimum d'anticipation ». (H., président d'association, recherche 1)

Les patients qui ont des savoirs aboutis de cet ordre utilisent le vocabulaire consacré dans le domaine en question, ce qui est logique car les concepts scientifiques se transmettent par le langage (Vygotski, 1997).

Dans une de leur forme accomplie, les savoirs savants sont traduits en savoirs experts (Perrenoud, 1998 ; Johsua, 1998). Les savoirs experts ont pour sources de production les situations-problèmes (Cheneval Armand, Ginestié, 2009). C'est ainsi que certains patients s'engagent pour développer des solutions techniques qui répondent aux besoins qu'ils ont pu constater. Ces patients agissent comme des «prosumers» (Ritzer, Jurgenson, 2010), soit comme des consommateurs qui produisent des produits pour leur propre besoin, voire même plutôt comme des «lead-users » (Becheur, Gollety, 2006) qui sont des prosumers qui mettent à disposition de leurs pairs le produit ainsi créé.

\section{III- Discussion}

\section{Limites de l'étude et de l'analyse}

Faire parler le terrain est un art autant qu'une science (Levi-Strauss, 1962). La proximité des auteurs avec leur sujet et la durée de recueil des données, sur une période de dix ans, a facilité l'analyse. La réalité capturée semble correspondre aux faits. Néanmoins, cette étude conceptuelle et exploratoire gagnerait à être reproduite. Ceci devrait être fait en tenant compte des résultats obtenus qui indiquent l'importance de réaliser les études auprès de différents profils de patients, profils déterminés à partir de leur niveau d'engagement au service de la collectivité et de l'élaboration plus ou moins collective de leurs savoirs. 


\section{L'inscription de ces savoirs dans le champ de la complexité et dans le rapport savoirs/pouvoirs}

Théoriquement, on peut discuter si l'accès à l'expérience d'autrui qui facilite le passage des savoirs implicites aux savoirs explicites demeure de l'ordre d'un savoir expérientiel, si le fait que les savoirs situés qui se construisent en réponse aux besoins constatés dans les expériences collectives empruntent majoritairement à l'expérience ou aux connaissances des normes exogènes qui permettent de statuer sur le caractère injuste des situations, ou à un esprit critique qui a le potentiel de les libérer des dogmes. Comme la théorie a besoin de l'expérience, les savoirs expérientiels ont besoin de se frotter aux savoirs savants (Dewey, 1938 ; Vygotski, 1997). Les savoirs de l'expérience peuvent en effet profiter des savoirs introduits par le haut : ainsi, l'expérience vécue gagne à être confrontée à certaines normes, en particulier à celles de l'ordre de l'éthique ou du droit (Nussbaum, 2001 ; Panet 2008). C'est parce que le consentement aux soins est un droit que certaines pratiques peuvent être remises en question. Mais encore faut-il connaître les droits. D'où l'importance de la transmission de savoirs formels, y compris dans le cadre de pédagogies émancipatrices et dans l'élaboration des savoirs situés.

Certains patients accumulent des savoirs expérientiels, des savoirs situés et des savoirs savants qui interagissent entre eux comme avec l'environnement social. Leur vulnérabilité est devenue une force (Barrier, 2010), y compris sur le plan cognitif au point que certains patients soient animés par une passion cognitive (Gross, Gagnayre, 2014b) qui les pousse à produire des savoirs émancipateurs (Roux et al., 2009) en ce sens qu'ils questionnent les savoirs (et savoir-faire) introduits par le haut. Sans doute en effet que ce qui différencie le plus ces savoirs entre eux, ce n'est pas tant leurs processus de production, que leurs manifestations et leurs objectifs. Si continuum il y a, c'est d'ailleurs au niveau de ces dernières dimensions et non au niveau des savoirs eux-mêmes. Les savoirs expérientiels implicites diffèrent des savoirs expérientiels explicites car les premiers visent l'adaptation des modes de fonctionnement individuels (Chevrier, Charbonneau, 2000) tandis que les seconds visent une hétéro-adaptation c'est à dire une influence sur autrui pour que lui s'adapte en fonction d'eux. Les savoirs situés pour leur part visent à compléter les savoirs professionnels et académiques en interrogeant la réalité autrement que les acteurs traditionnels, tandis que les savoirs experts sont utilisés pour enrichir les autres savoirs et/ou pour agir directement sur le monde de la santé. Aussi y-aurait-il, d'après nos résultats, un lien entre les modalités d'émergence des savoirs et les pouvoirs qu'ils facilitent, ce qui amorcerait une explication des liens itératifs entre Savoirs et Pouvoirs. Si le lien entre le savoir et le pouvoir a déjà été souligné (Foucault, 1975), souligner que ces liens sont itératifs, c'est rappeler que les quatre types de savoirs identifiés se construisent et se valident en fonction des pouvoirs d'action ou d'influence qu'ils permettent d'acquérir sur la vie quotidienne, sur les autres et le monde. La vérité sert non seulement à organiser le monde, mais aussi à prendre du pouvoir sur lui, ce qui peut aller bien au-delà d'une simple maîtrise cognitive puisque cela peut aller jusqu'à penser et participer à sa reconfiguration. Ainsi, comme cela a été montré par ATD Quart Monde, les savoirs introduits par le bas (soit les savoirs expérientiels ou situés des patients), peuvent, s'ils sont invoqués, tenir le rôle de «savoirs d'émulation » afin d'être des «ballons d'oxygène » (Wresinsky cité par Roy, 2016) qui ont le pouvoir de fertiliser les savoirs dominants. 


\section{De l'utilité de tous ces types de savoirs pour améliorer la qualité des soins : l'exemple de la formation médicale}

Il serait possible de discuter l'apport de nos résultats dans divers secteurs d'activité des patients mais celui que nous prenons est celui de la formation médicale dans la mesure où nous expérimentons ce type d'application au quotidien au sein de notre université.

Se pose avec une grande acuité la question du profil des patients à intégrer comme enseignants, formateurs, auprès des étudiants en médecine, y compris dans des pays où leur engagement dans la formation médicale est plus courant (Gutteridge, Dobbins, 2009). Ce champ d'intervention des patients s'institutionnalise en France où, dans la lignée d'une expérimentation (Gross et al. 2017a, 2017b), de nombreuses équipes pédagogiques se saisissent de ce thème. Comme dans de nombreux autres espaces propices à l'engagement des patients, les équipes sont tiraillées entre leur souhait de recruter des «vrais patients » représentatifs de ceux que les médecins voient en consultation et celui d'établir des référentiels de compétences exigeants qui leur semblent nécessaires pour tenir cette fonction pédagogique. De plus, on constate sur le terrain que les équipes pédagogiques hésitent à recruter les patients au sein des mouvements associatifs, sans doute par crainte des liens d'intérêt avec l'industrie du médicament, ou par peur d'intégrer des savoirs militants au sein de l'institution universitaire. Ce qui les conduit à privilégier le recrutement de patients coupés de ces mouvements, et partant de là de patients auxquels il devient aisé de reprocher de porter des revendications individuelles, ou des perspectives singulières, sans intérêt pour les étudiants. Les orientations proposées visent à éviter l'effet «âne de Buridan » susceptible d'intervenir dans ce contexte d'injonctions paradoxales.

À partir de ce qui précède, nous insistons sur le fait que si les patients ont leur place comme enseignants dans la formation médicale et paramédicale, c'est pour leurs savoirs (Lechopier, 2015) et que cela concerne tous les types de savoirs, comme dans tous les autres champs qui sont ouverts à l'engagement des patients. Cela revient à mettre en actes le paradigme de l'approche centrée sur le patient et cela permet de s'inscrire dans l'une des recommandations de la Déclaration de Vancouver qui incite à veiller à recruter comme enseignants ou formateurs une diversité de profils de patients (Towle et al. 2016).

Les savoirs situés émergent de manière privilégiée dans des collectifs qui peuvent être associatifs. De plus, les savoirs situés sont la condition de la représentativité dans le sens où ils expriment un point de vue collectif. Quand on veut éviter que des perspectives idiosyncrasiques ne s'expriment, c'est le type de savoirs qu'il convient de convoquer. Les patients qui ont des savoirs situés sont ceux que l'on peut recruter pour contribuer aux cours sur la relation de soins ou pour apporter la perspective des patients sur la qualité des soins. Cette dernière perspective est en effet tout à fait stabilisée : celle qui a été identifiée à partir des enseignements réalisés au sein de l'Ufr SMBH par des patients recrutés pour leurs savoirs situés (Gross et al, 2017b) résonne notamment avec celle qui est promue par les patients 
engagés dans l'amélioration de la qualité des soins aux Etats-Unis (Ferguson, Frydman, 2004). La résistance concernant l'appartenance des patients au milieu associatif est un frein aux modalités d'intervention qui nécessitent des savoirs de cet ordre, alors qu'ils sont les seuls savoirs représentatifs puisqu'il sont le fruit d'un processus lors duquel ils ont pu monter en généralité.

En revanche, l'émergence des autres types de savoirs ne dépendent pas de collectifs. Ceci est un argument supplémentaire pour multiplier les modalités d'implication des patients, car il s'agit de partir des missions pour établir le profil des patients à recruter, profil à établir en fonction de leurs savoirs. Et non pas de décider des profils en amont des missions. Et cela sans oublier que les patients, pas plus que les professionnels de santé, ne sont omniscients. Certains peuvent avoir des savoirs savants dans un domaine et des savoirs expérientiels dans un autre. Il convient donc, de réinterroger pour chaque sujet traité où se situent les personnes, tout en leur donnant des opportunités d'évolution. Ainsi, les savoirs expérientiels implicites des patients sont utiles pour entraîner les futurs soignants à mener des entretiens cliniques, comme dans le cas des « health mentors » (Towle, 2014). Quant aux patients qui ont des savoirs expérientiels explicites, ce sont eux qu'il convient de recruter pour témoigner auprès des étudiants de leur vécu expérientiel dans les quatre domaines identifiés, sachant que ce type d'interventions est très apprécié des étudiants (Turner et al. 2000). Enfin, certains patients, sous condition d'avoir des savoirs savants, spécialisés, peuvent également participer à l'ingénierie pédagogique (Pomey et al. 2015a) et contribuer à l'enseignement de notions théoriques (Renard et al. 2014).

\section{Conclusion}

Les patients sont des sujets épistémiques produits par leur expérience de la maladie, expérience qui pour nombre d'entre eux est mise en lien avec celles de leurs pairs, et pour certains par des savoirs collectivement construits, voire confrontés aux savoirs savants ou professionnels. Ces savoirs, lorsqu'ils sont convoqués dans le système de santé, contribuent à le transformer. Sous réserve que les espaces qui les accueillent soient réellement participatifs, les régimes de vérité qui en découlent peuvent devenir plus démocratiques. Sachant que dans le cadre de ces régimes, «la vérité est liée circulairement à des systèmes de pouvoir qui la produisent et la soutiennent, et à des effets de pouvoir qu'elle induit et qui la reconduisent » (Foucault, 1977). Afin d'éviter un réductionnisme médical (Dalgalarrondo, 2008) qui soit totalisant, voire autocratique, son régime de vérité devrait systématiquement être co-construit avec tous ceux qui le vivent.

La taxinomie proposée contribue aux travaux conceptuels sur les savoirs profanes et l'intégration des acteurs issus de la société civile dans les actions portées par les professionnels. Elle permet aussi de déterminer quels profils recruter à quelle tâche. Dans la santé, il s'agit de recruter des patients, non pas en fonction de leur statut, de leur expérience de la maladie, ou de quelque autre critère, mais en fonction de leurs savoirs afin qu'ils participent à l'amélioration de la qualité des soins. Afin de multiplier les sources de rétroaction, il s'agit de concevoir différents types de sollicitations de manière à recruter 
différents profils de sujets épistémiques. Procéder ainsi est vertueux et équitable puisque c'est considérer que leur contribution est utile dans le cadre d'une médecine participative, quels que soient leurs types de savoirs. Certains de ces savoirs sont idiosyncrasiques, tandis que d'autres ont une portée plus générale et ont le potentiel d'enrichir les savoirs académiques et/ou professionnels. Tous visent à soutenir, en contrepoint à la vulnérabilité initiale liée à la maladie, les pouvoir d'agir individuels ou communautaires, de manière à influer sur la santé individuelle, celle d'autrui, ou sur la qualité du système de santé.

\section{Bibliographie}

Alinsky, S. (1946). Reveille for Radicals. Chicago: University of Chicago Press.

Akrich, M.\& Meadel, C. (2009). «Les échanges entre patients sur Internet ». La Presse Médicale, p. 1484-1493

Barrier, P. (2010). La blessure et la force. Paris : PUF.

Barrier, P. (2012). «Éclairage sur les processus d'auto-normativité dans la démarche d'accompagnement et d'éducation des équipes soignantes », Recherche en soins infirmiers, $\mathrm{n}^{\circ} 110$, p. 7-12.

Blondiaux, L. (2008). «Le profane comme concept et comme fiction politique », in T. Fromentin \& S. Wojcik, Le profane en politique, Paris : l'Harmattan, p. 37-51

Bravo et al. (2015). «Conceptualizing patient empowerment : a mixed method study ». BMC Health Services Research, 15:252

Chevrier, J.\& Charbonneau, B. (2000). «Le savoir-apprendre expérientiel dans le contexte du modèle de David Kolb ». Revue des sciences de l'éducation, 26(2), 287-324.

Compagnon, C. \& Ghadi, V. (2014). « Pour l'An II de la Démocratie sanitaire ». Rapport remis au ministère des Affaires sociales, de la Santé et des Droits des femmes.

Dalgalarrondo, S. (2008). «Quand le malade ne correspond pas... »(Commentaire). Sciences sociales et santé, Volume 26/2, p. 33-38

D'Arripe, A. \& Routier C. (2013). «Au-delà de l'opposition entre savoir profane et savoir expert : une triangulation des méthodes » Recherche qualitative, HS 15, p.221-234

Dalgalarrondo (2008) «Quand le malade ne correspond pas... »(Commentaire). In: Sciences sociales et santé. Volume 26, n², 2008. pp. 33-38.

Demailly, L. (2015). Les médiateurs de santé pairs en santé mentale, entre expérience, technique et style, Sciences et Actions Sociales, ${ }^{\circ} 1$, pp. 1-22

Desroche, H. (1990). Entreprendre d'apprendre, d'une autobiographie raisonnée aux projets d'une rechercheaction. Paris : Éditions ouvrières.

Dewey, J. (1938). Expérience et éducation. Paris, Armand Colin.

D’Ivernois, JF. \& Gagnayre, R. (2001). « Mettre en œuvre l'éducation thérapeutique ». ADSP, n³6, p.11-13.

Druais, PL. (2015). «La place et le rôle de la Médecine Générale dans le système de santé » Rapport réalisé à la demande de la ministre de la santé.

Ferguson, T. \& Frydman, G. (2004). "The first generation of e-patients: These new medical colleagues could provide sustainable healthcare solutions”. BMJ : British Medical Journal. 328(7449):1148-1149. 
Foucault, M. (1975). Surveiller et punir, Paris : Gallimard.

Foucault, M. (1977). Dits et Écrits II 1954-1988, Paris : Gallimard.

Freire, P. (2001). Pedagogia da indignação: cartas pedagógicas e outros escritos. Interface - Comun Saúde Educ.

Galvani, P. (2016). Conscientiser les moments décisifs (kaïros) de la pratique, une voie de recherche réflexive Actes du colloque Repaira.

Galvani, P. (1999). «Fertilisation croisée des savoirs et ingénierie d'alternance socio-formative. Le programme de recherche-formation-action Quart Monde/Université ». Revue française de pédagogie, ${ }^{\circ}$ 128, 25-34

Gardien, E. (2010). La pairémulation dans le champ du handicap : Histoire, pratiques et débats en France.

Rhizome, pp.3-4. <hal-00673245>

Gourgues et al. (2013). «Gouvernementalité et participation. Lectures critiques », Participations, Vol. 2, n6, p. $5-33$.

Gross, O. (2017). L'engagement des patients au service du système de santé, Rueil Malmaison : Doin.

Gross, O \& Gagnayre, R. (2014). "What patients report that they do in the french health care system and the competencies and personality traits required". Therapeutic Patient Education, 6 (2) 20104

Gross, O. \& Gagnayre, R. (2014b). "Le rôle de la passion dans la logique de l'agir des patients-experts", In Jouet, E. \& Las Vergnas, O. \& Noelle-Hureaux, E., (dir), Nouvelles interventions réflexives dans la recherche en santé: savoirs expérientiels des malades et formation par la recherche en sciences infirmières, Paris, Editions des Archives Contemporaines.

Gross, O. \& Sannié, T. \& Traynard, PY. \& Gagnayre, R. (2016). « Scientifiser son malheur : discussion sur le caractère émancipatoire d'une formation des patients intervenant dans l'éducation thérapeutique », Recherches \& Educations, pp.114-128

Gross, O.\& Ruelle, Y.\& Sannié, T., Khau, C-A.\& Marchand, C.\& Cartier, T.\& Mercier, A.\& Gagnayre, R. (2017a). «Un département universitaire de médecine générale au défi de la démocratie sanitaire : l'engagement de patients-enseignants ». Revue des Affaires Sociales, vol. 1, p. 61-78

Gross, O. \& Ruelle, Y.\& Khau, CA. \& Sannié, T.\& Gagnayre, R. (2017b). «L'utilité des patients-enseignants dans la formation initiale des médecins généralistes ». Éducation Santé Sociétés, Vol. 3, n², p. 37-54

Gross, O. \& De Andrade, V. \& Gagnayre, R. (2017c). "Pratiques et apport des recherches communautaires en éducation thérapeutique : une revue de littérature ». Santé Publique, vol. 29, n4, p. 551-562

Gutteridge, R. \& Dobbins, K. (2009). "Service user and carer involvement in learning and teaching: A faculty of health staff perspective", Nurse Education Today, Vol. 30, p. 509-514

Haas, P. (1992). "Introduction: epistemic communities and international policy coordination". International Organization, 46(1), p. 1-35.

Haraway, D. (1991). Situated Knowledges » in Simians, Cyborgs and Women. The Reinvention of Nature. Londres: Free Association Books. • • •

Hardey M. (1999). "Doctor in the house: the Internet as a source of lay knowledge and the challenge to expertise". Sociology of Health \& Illness, Vol. 21, $n^{\circ} 6$, p. 820-835

Jankelevitch, V. \& Berlowitz, B. (1978). Quelque part dans l’inachevé, Paris, Gallimard.

Jouet, E. \& Flora, L.\& Las Vergnas, O. (2010). Construction et reconnaissance des savoirs expérientiels des patients : Note de synthèse. Pratiques de Formation, p.13-94 
Kolb, D.A. (1984). Experiential learning : experience as the Source of Learning and Development. NJ: Prentice Hall.

Lechopier, N. (2015). « Participation des patients à la formation médicale », Rhizome, vol.4, n 58, p. 15-16.

Le Boterf, G. (2003). Construire les compétences individuelles et collectives. Paris : Eyrolles.

Lévi-Strauss C. (1962). La Pensée sauvage. Paris : Plon.

Mac Laughlin, H. (2009). «What's in a Name: 'Client', 'Patient', 'Customer', 'Consumer', 'Expert by Experience', 'Service User’—What's Next? » The British Journal of Social Work, vol. 39, 6 (1), p. 1101-1117

Massé, R. (2005). «Les fondements éthiques et anthropologiques d'une participation du public en santé publique ». Éthique publique, vol. 7, no 2, p. 107-124.

Morin, E. (1980). La Méthode : la Vie de la vie (tome 2), Paris : Le Seuil.

Nussbaum MC. (2001). Women and Human Development: The Capabilities Approach. Cambridge: Cambridge University Press.

NHS. (2014). Public involvement in research applications to the National Research Ethics Service. Comparative analysis of 2010 and 2012 data. INVOLVE, Health Research Authority.

Panet S. \& Duray-Soundron C. (2008). «C’est comme ça que ça germe. Changement social au Sénégal : le cas de l'ONG Tostan ». In: Dubois J-L, Repenser l'action Collective. Paris : l'Harmattan.

Pereira Paulo, L. \& Tourette-Turgis, C. (2014). « De l'accès à l'expérience des malades à la professionnalisation de leurs activités : reconnaître le care produit par les malades ». Le sujet dans la cité, vol.2, n5, p. 150-159.

Perrenoud, P. (1998). «La transposition didactique à partir de pratiques : des savoirs aux compétences ». Revue des sciences de l'éducation, vol. 24, n³, p. 487-514

Piaget, J. (1970). Psychologie et épistémologie. Paris : Editions Gonthier.

Piaget J. (1974). Réussir et comprendre. Paris : PUF.

Pineau, G. (1986). Temps et contretemps en formation permanente. Paris, Éditions universitaires UNMFREO.

Pomey et al. (2015a). «Le Montréal Model : enjeux du partenariat relationnel entre patients et professionnels de la santé ». Santé Publique, HS (S1) p.41-50

Pomey et al. (2015b) "Patient partnership in quality improvement of healthcare services: patients' inputs and challenges faced," Patient Experience Journal, vol. 2: n 1 , Article 6.

Romeyer, H. (2008). «TIC et santé : entre information médicale et information de santé», Revue tic\&société vol. $2, \mathrm{n}^{\circ} 1, \mathrm{p} .27-44$

Renard, E. \& Alliot-Licht, B. \& Gross, O. \& Roger Leroi, V. \& Marchand, C. (2014). «Study of the impacts of patients-educators on the course of basic sciences in dental studies". European Journal of Dental Education, vol. 19-1, p. 31-37.

Renault E. (2004). L'expérience de l'injustice. Paris : La Découverte.

Ritzer, G. \& Jurgenson, N. (2010). «Production, consumption, prosumption: the nature of capitalism in the age of the digital 'prosumer' ». Journal of Consumer Culture, vol.10, $\mathrm{n}^{\circ} 1$, p. 13-36

Roux, J. et al (2009). "Les « passions cognitives » ou la dimension rebelle du connaître en régime de passion ». Revue d'anthropologie des connaissances, vol. 3, n`3, p. 369-385 
Roy, A. (2016). «Joseph Wresinski : pionnier de l'empowerment radical made in France », Mouvements, vol. 1, $\mathrm{n}^{\circ} 85$, p. $87-94$.

Santi, P. «Catherine Tourette Turgis, du côté des vivants ». Le Monde du 6 avril, http://www.universitedespatients.org/wp-content/uploads/2016/04/Catherine-Tourette-Turgis-article-

LeMonde_05_04_16.pdf

Sackett et al. (1996). «Evidence based medicine: what it is and what it isn't”. BMJ, p. 312-371

Stehr, N. (2000). « Le savoir en tant que pouvoir d'action ». Sociologie et sociétés, vol. 32, n 1 , p.157-170.

Tardieu, G. (2015). « Coconstruction de savoir à l'université populaire Quart Monde », Ecologie \& politique, vol.2, $\mathrm{n}^{\circ} 51$, p. 81-92.

Tourette-Turgis, C. (2015). L'éducation thérapeutique : la maladie comme occasion d'apprentissage, Paris : De Boeck.

Towle, A. (2014). "The expert patient as teacher: an interprofessional health mentors programme". The Clinical Teacher, $\mathrm{n}^{\circ} 11$, p. 301-306

Towle et al. (2016). "The patient' voice in health and social care professional education. The Vancouver Statement”. International Journal of Health Governance, vol. 21, n 1 , p. 18-25

Turner, P. \& Sheldon, F.\& Coles, C.\& Mountford, B.\& Hillier, R. \& Radway, P.\& Wee, B. (2000). "Listening to and learning from the family carer's story: an innovative a, roach in interprofessional education". Journal of Interprofessional Care, vol. 14, n 4 , p. 387-395.

UNESCO (2005). Vers les sociétés du savoir. Paris : Editions UNESCO.

Varela, F. J., Thompson, E., Rosch, E. (1993). L'inscription corporelle de l'esprit : sciences cognitives et expérience humaine. Paris : Seuil.

Vygotski, L. (1997). Pensée et langage. Paris : La Dispute. 